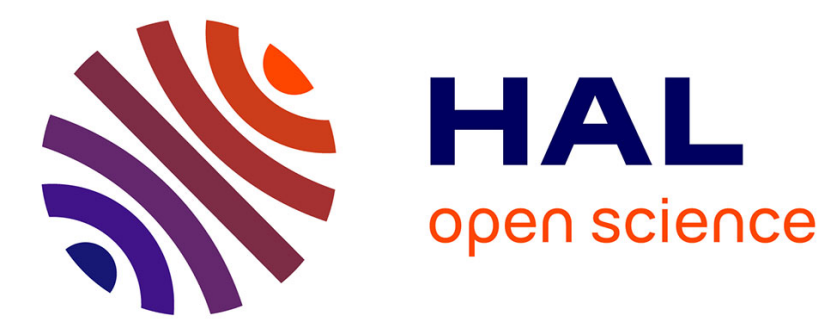

\title{
La Roumanie à Paris: exil politique et lutte anti-communiste
}

Béatrice Scutaru

\section{To cite this version:}

Béatrice Scutaru. La Roumanie à Paris: exil politique et lutte anti-communiste. Histoire@Politique : revue du Centre d'histoire de Sciences Po, 2014, 23, pp.1-11. hal-01157379

\section{HAL Id: hal-01157379 \\ https://hal.science/hal-01157379}

Submitted on 27 May 2015

HAL is a multi-disciplinary open access archive for the deposit and dissemination of scientific research documents, whether they are published or not. The documents may come from teaching and research institutions in France or abroad, or from public or private research centers.
L'archive ouverte pluridisciplinaire $\mathbf{H A L}$, est destinée au dépôt et à la diffusion de documents scientifiques de niveau recherche, publiés ou non, émanant des établissements d'enseignement et de recherche français ou étrangers, des laboratoires publics ou privés. 
Béatrice Scutaru, « La Roumanie à Paris : exil politique et lutte anti-communiste », Histoire@Politique. Politique, culture, société, n² 23, mai-août 2014 [en ligne, www.histoire-politique.fr]

\title{
La Roumanie à Paris : exil politique et lutte anti-communiste
}

\author{
Beatrice Scutaru
}

«La liberté à l'Ouest » est le choix fait par de nombreux Roumains à la suite de l'avènement du communisme ${ }^{1}$. Plusieurs vagues d'émigration peuvent être identifiées jusqu'en 1989, entraînées par les évolutions internes de l'État roumain ainsi que par les changements produits au niveau international. Les publications traitant de ce sujet font une distinction entre l'exil littéraire et l'exil politique. J usqu'à présent, c'est surtout ce premier qui a suscité l'intérêt des chercheurs ${ }^{2}$. De manière surprenante, l'étude de l'action politique est plutôt laissée de côté, à l'exception de cas particuliers de dissidents roumains très médiatisés, comme Paul Goma ${ }^{3}$. Sont publiés notamment des documents d'archives et des dossiers réalisés par la Securitate (police secrète roumaine), des récits et des entretiens ${ }^{4}$. Pour cette raison, notre intérêt se porte tout particulièrement sur ce qu'on appelle l'exil politique ou l'exil actif, engagé, traité ici dans le sens que lui donne Stéphane Dufoix: "l'espace relationnel des groupes luttant politiquement contre le régime en place dans leur pays d'origine ${ }^{5}$ ». En effet, c'est sur les Roumains qui luttent contre le régime communiste que va se concentrer cette étude et principalement sur ceux qui décident de s'installer à Paris. Le choix de nombreux dissidents se porte sur la « capitale des libertés » en raison de son image de lieu d'accueil et d'asile pour les citoyens fuyant les dictatures ${ }^{6}$. De plus, Paris tient une place importante dans la tradition de la vie politique et culturelle roumaine. C'est ici que se sont formés les jeunes roumains qui, au XIX ${ }^{\mathrm{e}}$ siècle, ont lutté pour la réalisation des grands idéaux nationaux, comme l'unité et l'indépendance nationales. Un autre élément qui explique la concentration des exilés en région parisienne est la proximité des institutions françaises s'occupant des réfugiés, les possibilités plus importantes de trouver un emploi et l'existence de communautés nationales déjà organisées. Ainsi, dans les années 1970et 1980, Paris devient « un pôle actif de soutien à la dissidence du bloc soviétique ${ }^{7}$ ».

Notre propos ne se veut pas un aperçu monographique exhaustif des organisations, associations et personnalités en exil mais - à travers quelques exemples - il s'agit d'illustrer l'importance de l'exil politique roumain dans la lutte anti-communiste.

\footnotetext{
${ }^{1}$ Cette expression - « choisir la liberté » - est régulièrement employée par les Roumains qui choisissent de quitter la Roumanie pendant la guerre froide. (Sanda Stolojan, Au balcon de l'exil roumain à Paris avec Cioran, Eugène Ionesco, Mircea Eliade, Vintila Horia, Paris, l'Harmattan, 1999, p. 345).

2 Eva Behring, Scriitori romani în exil, 1945-1989, Bucarest, Editura Fundatiei Culturale, 2001.

3 Catherine Durandin, « Le Paris de Paul Goma », dans Antoine Marès, Pierre Milza (dir.), Le Paris des étrangers depuis 1945, Paris, Publications de la Sorbonne, 1994, p. 169-188.

4 Dinu Zamfirescu, Si noi am condamnât comunismul din exilul parizian, Bucarest, Paideia, 2008.

${ }^{5}$ Stéphane Dufoix, « Les légitimations politiques de l'exil », Genèses, vol. 34, 1999, p. 53.

6 Ion Calafeteanu, Politica si exil. Din istoria exilului romanesc 1946-1950, Bucarest, Editura Enciclopedica, 2000, p. 38.

${ }^{7}$ Antoine Marès, Pierre Milza (dir.), Le Paris des étrangers..., op. cit., p. 10-14, 134-138.
} 
Béatrice Scutaru, « La Roumanie à Paris : exil politique et lutte anti-communiste », Histoire@Politique. Politique, culture, société, n²3, mai-août 2014 [en ligne, www.histoire-politique.fr]

Leur choix permet de dégager les principales formes d'organisation ainsi que les fonctions de l'exil. Pour la réalisation de cette étude ont été consultés notamment les archives de la Ligue de défense des droits de l'homme en Roumaine, ainsi que les dossiers personnels des exilés, conservés à l'Institut d'investigation des crimes du communisme et de la mémoire de l'exil roumain (IICCMER) ou réalisés par la police secrète roumaine (Centre national pour l'étude des archives de la Securitate).

\section{Le choix de la liberté à l'Ouest}

L'émigration roumaine à l'Ouest de l'Europe connaît plusieurs étapes, avec une première période importante à la fin des années 1940 et une autre culminant durant les décennies 1970-1980. L'étape comprise entre 1946 et 1956 a un caractère principalement politique et concerne surtout des intellectuels, des étudiants et des hommes politiques. Suite à l'abdication du roi Michel (1947), le nouveau régime demande à tous les citoyens roumains se trouvant à l'Ouest de revenir en Roumanie. Beaucoup d'entre eux refusent et décident de s'installer en Occident. Appartiennent également à cette première vague les Roumains qui, après s'être compromis avec les régimes ayant collaboré avec l'Allemagne nazie, choisissent la voie de l'exil, notamment en Espagne ${ }^{8}$. La défaite de la révolution magyare (1956) marque la fin de cette première période. Les Roumains comprennent que la soviétisation est un phénomène de longue durée et que les Occidentaux n'interviendront pas pour libérer les pays situés à l'Est de l'Europe. C'est donc dans la durée que les exilés doivent désormais envisager l'exil et leur lutte contre le communisme ${ }^{9}$.

La période suivante, qui dure jusqu'à la fin des années 1960, est plus difficile à définir. Selon Monica Lovinescu, une importante figure de l'exil roumain à Paris, elle est principalement constituée de Roumains quittant le pays pour des raisons économiques, souhaitant refaire leur vie en Occident ${ }^{10}$. Cependant, il s'avère difficile d'établir une distinction nette entre l'émigration « politique » qui relève de l'exil et l'émigration « économique ». Plusieurs études ont montré qu'il est tout à fait possible que les positions de départ des exilés changent au fil du temps ${ }^{11}$. D'une part, l'exil peut politiser une vie : les immigrés considérés comme « économiques » peuvent, une fois arrivés en Occident, s'impliquer dans la lutte anti-communiste ${ }^{12}$. D'autre part, lorsqu'ils arrivent à l'Ouest, certains militants politiques choisissent d'abandonner leur combat. « Le rapport des réfugiés à la politique n'est ni obligé, ni statique, il n'est

\footnotetext{
8 Antoine Marès, « Exilés d’Europe centrale de 1945 à 1967 », dans Antoine Marès, Pierre Milza (dir.), Le Paris des étrangers ...op. cit., p. 140-141.

9 Eva Behring, Écrivains roumains en exil, 1945-1989 [Écrivains roumains en exil, 1945-1989], Bucuresti, Editura Fundatiei Culturale Romanie, 2001, p. 29.

10 Monica Lovinescu, «Dialoguri pe unde scurte», dans Gheorghe Glodeanu, Incursiuni în literatura diasporei si a disidentei, Bucuresti, Libra, 1999, p. 303-314.

${ }^{11}$ Frithjof Trapp, «L'exil en tant que résistance. Ébauche d'une typologie », dans Gilbert Krebs et Gérard Schneilin (dir.), Exil et Résistance au national-socialisme 1933-1945, Paris, Université de la Sorbonne Nouvelle, Publications de l'Institut d'allemand, 1998, p. 51.

12Emmanuelle Loyer, « Exile», dans Akira Irie, Pierre-Yves Saunier (dir.), The Palgrave Dictionary of Transnational History, Hampshire, New York, Basingstoke, Palgrave Macmillan, 2009, p. 368. Geneviève Dreyfus-Armand, L'exil des républicains espagnols en France de la guerre civile à la mort de Franco, Albin Michel, 1999, p. 424.
} 
Béatrice Scutaru, « La Roumanie à Paris : exil politique et lutte anti-communiste », Histoire@Politique. Politique, culture, société, n²3, mai-août 2014 [en ligne, www.histoire-politique.fr]

pas non plus prévisible ${ }^{13} »$. Cette période est également caractérisée par une fluctuation du nombre de départs à l'étranger, comme le montre le graphique suivant :

Graphique n¹: Évolution de l'émigration roumaine de 1957 à 1989

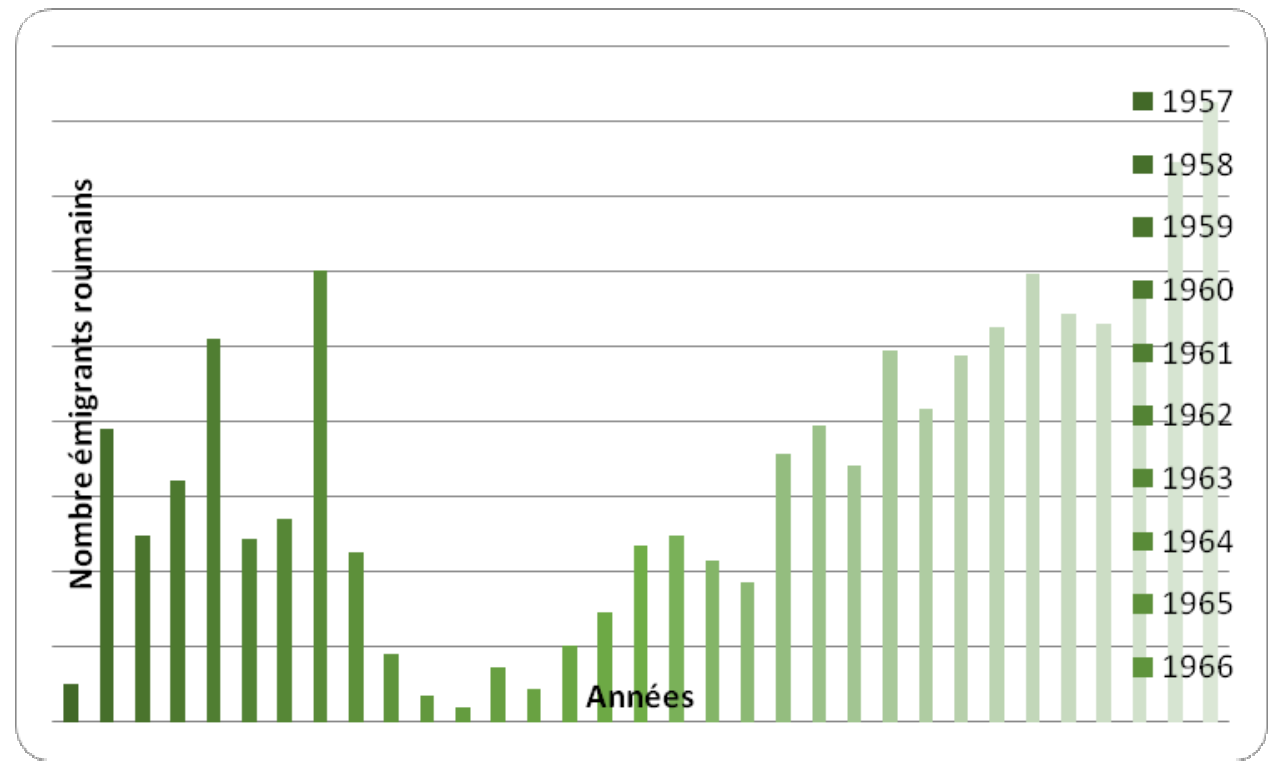

Source : Istvan Horvath, Remus Gabriel Anghel, "Migration and Its Consequences for Romania”, Südosteuropa, 2009, n 57, p. 387.

Une des principales difficultés est d'établir le nombre exact de Roumains ayant choisi de s'installer en France. Les données disponibles concernent surtout l'évolution générale de l'émigration roumaine. Le graphique renseigne sur les départs des Roumains à l'étranger après la première période d'émigration identifiée dans cette étude. Après une évolution fluctuante - mais concernant un nombre important de départs - la deuxième moitié des années 1960 est caractérisée par une baisse, principalement en raison de l'image positive de Nicolae Ceausescu. La relative ouverture du régime tant sur le plan de la politique intérieure qu'extérieure, l'opposition à l'invasion de la Tchécoslovaquie, donnent de l'espoir aux Roumains. Cependant, le durcissement de la politique interne amorcé par Nicolae Ceausescu en $1971^{14}$ déclenche une nouvelle hausse des départs. Ces mesures diminuent l'enchantement produit par la prise de position du chef de l'État roumain contre l'invasion de la Tchécoslovaquie. La troisième vague débute dans les années 1970, allant jusqu'en 1989. Cependant, alors que les demandes d'asile formulées par les citoyens roumains augmentent, il devient de plus en plus difficile pour les Roumains de partir à l'étranger. La signature de l'acte final d’Helsinki n'améliore en rien la situation. Au contraire, comme le souligne à plusieurs reprises l'ambassadeur

\footnotetext{
${ }^{13}$ Ioana Popa, « Dépasser l'exil. Degrés de médiation et stratégies de transfert littéraire chez les exilés de l’Europe de l'Est en France », Genèses, 2000/1, n 38, p. 32.

${ }^{14}$ Nicolae Ceausescu annonce, le 6 juin 1971, un renforcement du contrôle du Parti sur l'action des intellectuels qui entrent en contact avec la population et qui doivent prendre part activement à la création del'homme nouveau, del'homo comunistus.
} 
Béatrice Scutaru, « La Roumanie à Paris : exil politique et lutte anti-communiste », Histoire@Politique. Politique, culture, société, n²3, mai-août 2014 [en ligne, www.histoire-politique.fr]

français en Roumanie, il est encore plus difficile qu'au préalable de quitter la Roumanie. Après 1975, cela devient un véritable parcours du combattant «long et compliqué ». Certaines personnes doivent même renouveler pendant plusieurs années les procédures administratives de demande de visa, sans recevoir de réponse positive $^{15}$. Cela ne signifie pas que les Roumains ont renoncé à l'idée de s'installer en Occident. Ils décident juste de contourner le système traditionnel et les tentatives de passage clandestin de la frontière se multiplient. Malgré les contraintes imposées et les campagnes de presse menées, le régime de Bucarest ne réussit donc pas à empêcher ses citoyens de quitter le pays, comme l'illustre le graphique $\mathrm{n}^{\circ} 1$.

\section{Exister et résister à Paris}

D’ailleurs, la présence d'émigrés à l'Ouest symbolise une «défaite » pour le gouvernement de Bucarest. En quittant leur pays, les Roumains « votent avec les pieds » contre le régime communiste ${ }^{16}$. En choisissant la voie de l'exil, ils passent du statut de citoyens d'un État, qui bénéficient, même dans une dictature, de certains droits et d'un minimum de protection, à celui de « réfugiés », perdant ainsi leurs possessions, leur métier, leur situation sociale et politique. Le départ en exil est alors perçu comme un acte de résistance, mettant en question la légitimité du gouvernement communiste roumain ${ }^{17}$.

Les exilés affirment représenter la «vraie» Roumanie, la «véritable » nation roumaine. Une dissociation est alors opérée entre la nation, le peuple et l'État: la nation n'est plus sous l'autorité de l'État - elle est en exil. Ainsi se constitue l'« exopolitie», cet « espace-temps politique à la fois national et transétatique » fruit de la rupture du lien avec l'État d'origine et la négation de sa légitimité. Les exilés vivent alors une double expérience : ils ont accepté de couper les liens avec la patrie mais maintiennent une continuité avec elle par la mise en place d'actions, de mouvements nationaux politiques ou culturels à l'étranger ${ }^{18}$. Ils justifient ces actions par la loyauté envers leur « nation » et soutiennent être les véritables gardiens de la souveraineté nationale, la vraie voix du peuple ${ }^{19}$. Dans un discours tenu le 27 mars 1972, Constantin Cesianu ${ }^{20}$ affirme que « le seul privilège des exilés est celui de pouvoir faire connaître au monde la réalité, les vérités, que leurs compatriotes ne peuvent exprimer. C'est un devoir qu'ils doivent accomplir car ils défendent ainsi les intérêts supérieurs et permanents de la Roumanie ${ }^{21}$ ».

\footnotetext{
15 Centre des archives diplomatiques de Nantes (CADN), fonds Bucarest-ambassade, carton $n^{\circ} 405$, dépêche de l'ambassadeur de France en Roumanie pour le ministre des Affaires étrangères: l'émigration, Bucarest, 8 juillet 1976, 9 p.

16 Magdolina Barath, «Attempts to Create Unity in Hungarian Political Emigration after the 1956 Revolution", dans Peter Jasek (dir.), Anti-Communist Resistance in Central and Eastern Europe, Bratislava, Ustav Pamati Naroda, 2011, p. 798.

${ }^{17}$ Frithjof Trapp, « L'exil en tant que résistance..., op. cit., p. 45.

18 Stéphane Dufoix, « Sertorius ou Prospero ? », Socio-anthropologie [En ligne], 2001/9, mis en ligne le 15 janvier 2003, consulté le 15 novembre 2013, 5 p.

19Emmanuelle Loyer, « Exile »..., op. cit., p. 369.

${ }^{20}$ Ancien membre du PNL et prisonnier politique, Constantin Cesianu obtient en 1967 le droit de quitter définitivement la Roumanie pour la France.

${ }^{21}$ Institut d'investigation des crimes du communisme et de la mémoire de l'exil roumain, fonds Sanda Stolojan, dossier nº, l'activité de l'Institut universitaire roumain Charles Ier, septembre 1972, 6 p.
} 
Béatrice Scutaru, « La Roumanie à Paris : exil politique et lutte anti-communiste », Histoire@Politique. Politique, culture, société, n²3, mai-août 2014 [en ligne, www.histoire-politique.fr]

Les émigrés roumains tentent de dévoiler à l’opinion occidentale « le vrai visage de la Roumanie», la réalité concernant la politique intérieure menée par le régime communiste. Leurs actions sont également censées influer sur la politique roumaine de la France. Pour atteindre leurs objectifs, ils tentent d'agir tant auprès des citoyens et des médias, que des hommes politiques et des organismes internationaux. Néanmoins, afin d'agir et d'informer, les exilés doivent d'abord réussir à recueillir des informations sur les événements qui se déroulent en Roumanie. Plusieurs possibilités se présentent à eux. De nombreux dissidents n'hésitent pas à prendre contact directement avec les exilés, afin de les mettre au courant de leurs activités. Les nouveaux émigrés arrivés à Paris fournissent aussi des informations utiles qui alimentent les chroniques des émissions de radio ou les rapports fournis aux organismes internationaux de défense des droits de l'homme. Les exilés tentent également d'entrer en contact avec tous les Roumains se rendant à Paris - en voyage touristique, déplacement officiel, etc. - afin d'avoir des nouvelles. À son tour, la Securitateutilise ces rencontres pour obtenir des renseignements sur l'exil roumain : préoccupations professionnelles, relations entre les exilés, position occupée dans le cadre de l'émigration roumaine, activité politique, perception par les autorités françaises, position envers les émigrés roumains souhaitant visiter la Roumanie, etc. À partir de ces renseignements est décidée la démarche à suivre pour bloquer ou, pour le moins, limiter l'effet des manifestations de l'exil parisien ${ }^{22}$.

L'ambassade roumaine de Bucarest a aussi un rôle à jouer. Elle agit en direction de la communauté roumaine établie en France afin d'obtenir son soutien et de contrecarrer le discours anti-communiste des exilés politiques. Dès la fin des années 1950, les dirigeants roumains comprennent qu'afin d'améliorer les relations avec les pays occidentaux, ils doivent impérativement modifier la manière dont le pays est perçu. Cela passe, dès le début par une volonté de changer la manière dont la Roumanie est présentée dans les publications françaises, pour ensuite tenter de mobiliser de nombreux autres vecteurs (cinéma, télévision, associations...) et acteurs (exilés, lecteurs, touristes, etc. $)^{23}$.

La volonté d'avoir une emprise sur la diaspora roumaine est illustrée par la querelle autour de l'église roumaine de Paris. Un litige existe sur l'usage de cette église, propriété de l'État roumain depuis 1882, dont l'administration et l'usage des locaux sont confiés (1948) à une association animée par le père Vasile Boldeanu, réfugié en France depuis $1946^{24}$. L'église joue un rôle politique, devenant l'objet du conflit entre le régime communiste roumain et les dissidents installés à Paris. Les deux parties prennent conscience du potentiel de cette institution, son contrôle pouvant servir à asseoir une certainemainmise sur la communauté roumaine de Paris, ou au moins sur une grande partie de celle-ci. La diaspora roumaine ${ }^{25}$ peut devenir un groupe d'influence et un partenaire de dialogue avec les autorités françaises ${ }^{26}$.Après avoir

\footnotetext{
22 CNSAS, Monica Lovinescu, 349. L. Barladeanu, op. cit., p. 6-18.

23 Beatrice Scutaru, Les relations entre les sociétés française et roumaine des années 1960 à 1995 : un atout de l'ancrage de la Roumanie à l'Europe?, thèse de doctorat sous la direction d'Yves Denéchère, université d'Angers, 2013, p. 594.

${ }^{24}$ CADN, Bucarest, 470, note : Église de la rue J ean de Beauvais, 12 novembre 1976, 5 p.

${ }^{25}$ Le terme de « diaspora » est utilisé ici pour englober la totalité des Roumains habitant en France, en opposition à celui de « dissidence » qui concerne les opposants au régime communiste roumain.

26 Ovidiu Bozgan, « Biserica Ortodoxa Româna din Paris în primii ani postbelici », dans Ovidiu Bozgan (dir.), Studii de istoria Bisericii, Bucarest, 2000, p. 43-66.
} 
Béatrice Scutaru, « La Roumanie à Paris : exil politique et lutte anti-communiste », Histoire@Politique. Politique, culture, société, n²3, mai-août 2014 [en ligne, www.histoire-politique.fr]

longtemps accepté cette situation, en 1972, le nouvel ambassadeur roumain à Paris, Constantin Flitan, entreprend des nouvelles démarches afin de récupérer cette église. À plusieurs reprises, le litige est porté devant les tribunaux qui reconnaissent aux deux associations le droit de résider dans les lieux, ramenant la situation au statu quo ante, et rendant ainsi impossible l'expulsion du père Boldeanu par la voie judiciaire. Refusant d'accepter cette situation, les autorités roumaines déplacent la question sur un plan politiqueet insistent pour que le gouvernement français règle la question, par voie administrative ${ }^{27}$.Pour tenter de débloquer la situation, Bucarest multiplie les démarches sur le plan politique ${ }^{28}$ mais en vain, la situation restantinchangée jusqu'en 1989.

\section{La culture traverse le rideau de fer}

Une autre action qui fédère la communauté roumaine est la recréation à Paris de la Fondation universitaire roumaine Charles Ier, le 8 décembre 1950. Elle est née de la volonté de faire perdurer une tradition initiée par le roi Charles Ier un siècle auparavant (3 mai 1881) et dissoute par le régime communiste de Bucarest ${ }^{29}$. C'est la sauvegarde de la liberté d'expression et de pensée, «abolies [en Roumanie] où la culture ne peut s'exprimer que par les voies étroites et doctrinaires tracées par le parti communiste » qui est le moteur des actions de la Fondation. En plus de promouvoir la culture et l'identité roumaines à l'Ouest, ces actions doivent également contribuer à l'unité de « tous les Roumains libres ${ }^{30}$ ». J usqu'en 1974¹, c'est par l'encouragement des créations, des manifestations littéraires et artistiques roumaines que la Fondation envisage d'atteindre son objectif. Ses membres assurent également une veille de la presse écrite française au sujet de la Roumanie. Ils n'hésitent pas à contacter les journaux pour leur demander la rectification des informations qu'ils considèrent comme non conformes à la réalité, sans beaucoup de succès ${ }^{32}$.

La Fondation encourage et soutient également la publication de revues et d'ouvrages en roumain et en français. Les publications en français (Bulletin scientifique roumain, Études roumaines, etc.) doivent éveiller l'intérêt et la sympathie des Occidentaux pour le peuple roumain, ainsi que donner l'occasion de "sortir du "ghetto" de l'exil ${ }^{33}$ » en faisant connaître les créations roumaines à l'Ouest. Pour donner l'occasion aux écrivains en exil de s'exprimer dans leur propre langue, la Fondation assure également des publications en roumain, comme la revue Fiinta Romaneasca [L'Être roumain]. Cette activité n'est pas nouvelle, la première revue publiée par des membres de la diaspora roumaine remontant à 1850 (România viitoare [La Roumanie future]), aux Etats-Unis. Initialement, ces publications ont pour objectif d'apaiser la nostalgie du pays d'origine, mais à la suite de l'instauration

${ }^{27} \mathrm{CADN}$, Bucarest, 470, note de la Direction des affaires politiques : Église de la rue J ean-de-Beauvais, Paris, 6 décembre 1976, 4 p ; 474, note : Église roumaine de Paris, Paris, 8 janvier 1979, 4 p.

28 Des démarches sont faites auprès d'Alain Poher, Président français par intérim (mars 1974), de J ean Sauvagnargues, ministre des Affaires étrangères (août 1974), de Michel Poniatowski, ministre d’État, ministre de l'Intérieur (29 août 1974), de J acques Chirac, Premier ministre (juillet 1975).

${ }^{29}$ F. Manolescu, Enciclopedia..., op. cit., p. 323.

30 IICCMER, Sanda Stolojan, 6, information concernant l'activité de la Fondation en 1970, 1971, 4 p.

${ }^{31}$ En 1974 la Fondation est forcée de stopper ses actions en raison de problèmes financiers.

32 IICCMER, Sanda Stolojan, 6, information concernant l'activité de la Fondation en 1970, 1971, 4 p.

33 Entretien avec Dumitru Tepeneag cité dans Ioana Popa, « Dépasser l'exil..., op. cit., p. 27. 
Béatrice Scutaru, « La Roumanie à Paris : exil politique et lutte anti-communiste », Histoire@Politique. Politique, culture, société, n²3, mai-août 2014 [en ligne, www.histoire-politique.fr]

du régime communiste la plupart des revues de l'exil prennent alors un ton anticommuniste ${ }^{34}$. Étant, pour la majeure partie d'entre elles, rédigées en roumain, ces publications s'adressent exclusivement aux Roumains en exil, ne touchant que dans une très faible mesure le public français. Elles permettent de garder un lien entre les membres de la communauté et fournissent des informations sur divers événements ayant lieu en Roumanie, sur les actions de l'exil, sur les publications littéraires, etc. De même que la présence des exilés, ces publications peuvent être considérées comme une forme de résistance anti-communiste, de lutte contre les canons imposés par la censure roumaine.

Une autre fonction de l'exil roumain est donc celle d'informer à la fois l'opinion publique occidentale et les Roumains de France et de Roumanie. Le contrôle exercé par le régime roumain rend très difficile la circulation des informations relatives tant à ce qui se passe en Occident qu'en Roumanie. L'information doit donc traverser les frontières roumaines, arriver en Occident, pour retourner ensuite en Roumanie par la voie des ondes radio, surtout par l'intermédiaire des émissions de la Radio Free Europe(RFE).

Toutefois, il ne serait pas possible de parler de RFE sans mentionner Monica Lovinescu et Virgil Ierunca, les deux noms de l'exil roumain irrémédiablement liés à cette radio. Ils quittent la Roumanie dans les années 1940 et deviennent, après leur mariage en 1952, le couple le plus connu de l'exil roumain de Paris. Monica Lovinescu (1923-2008) obtient en 1947 une bourse de l'Institut culturel français qui l'amène à Paris où elle décide de rester à la suite de la venue au pouvoir du Parti travailleur roumain. Elle ne tarde pas à s'impliquer dans la lutte contre le régime communiste roumain ${ }^{35}$. De 1951 à 1975, Monica Lovinescu réalise des émissions littéraires et musicales pour la Radiodiffusion française et, à partir de 1962, elle collabore avec RFE, jusqu'en 1992. Avec Virgil Ierunca, elle anime, à partir de 1967, les émissions, « Thèses et antithèses à Paris » et «Actualités culturelles roumaines », fournissant des informations sur les parutions parisiennes et roumaines et les débats artistiques dans les pays européens. Ils souhaitent dénoncer « cette chape de plomb tombée sur leur pays ${ }^{36}$ » et deviennent un des principaux soutiens des dissidents et protestataires roumains dans la presse de l'exil par l'intermédiaire du poste de radio Europe libre ${ }^{37}$. Elle est « pour des centaines de milliers de Roumains, [...] la voix qui ose présenter, au micro d'Europe Libre, le vrai visage de la Roumanie communiste ${ }^{38} \gg$.

Virgil (Untaru) Ierunca (1920-2006) ${ }^{39}$ quitte la Roumanie pour la France en 1946. De 1952 à 1975, il est employé par la radiodiffusion française comme rédacteur de certaines émissions politiques et culturelles en roumain. En 1975, il commence sa collaboration avec RFE pour des émissions, seul (« Povestea vorbei [L’histoire de la

\footnotetext{
34 Ilie Rad (dir.), J urnalism românesc in exil si diaspora, Bucarest, Tritonic, 2012, p. 31-54.

35 Édith Lhomel, « Monica Lovinescu, la force d’une voix », Le Courrier des pays del'Est, 2008, n 1067, p. 109.

36 Édith Lhomel, «Opération Villages Roumains», Politique internationale, dossier spécial «La Roumanie au seuil de l'Union européenne », automne 2004, n 105, p. 110-111.

37 FlorinManolescu, Enciclopediaexilului literar românesc. 1945-1989, Bucarest, Compania, 2003, p. 454.

38 Iulia Vladimirov, Monica Lovinescu în documentele Securitatii. 1949-1989, Bucarest, Humanitas, 2012, p. 9, 18.

39 Virgil Ierunca est le pseudo de Virgil Untaru.
} 
Béatrice Scutaru, « La Roumanie à Paris : exil politique et lutte anti-communiste », Histoire@Politique. Politique, culture, société, n²3, mai-août 2014 [en ligne, www.histoire-politique.fr]

parole] ») ou avec Monica Lovinescu ${ }^{40}$. Durant ces émissions, sont également lus des textes censurés ou interdits en Roumanie, créant ainsi l'image d'un Occident accueillant où les écrivains sont véritablement appréciés, faisant rêver les Roumains qui vivent avec le poids de la censure et de la Securitate ${ }^{41}$. Ils contribuent ainsi à créer le mythe de l'Occident, où les citoyens peuvent s'exprimer librement, sans crainte ni contrainte. Le rôle de ces émissions ne doit cependant pas faire oublier les critiques qui sont également formulées à leur égard. Dans plusieurs de ses ouvrages, l'écrivain Virgil Tanase - comme d'autres exilés - soutient que « le politique prenait le pas sur la littérature. Il y avait une volonté délibérée et choquante de propagande anticommuniste et pro-occidentale. [...] Inconsciemment, les directeurs et journalistes de $\mathrm{RFE}$ étaient, en effet, des anticommunistes primaires, en leur âme et conscience, incapables d'une analyse dépassionnée ${ }^{42}{ }^{2}$.

\section{S'unir pour mieux dénoncer}

Très rapidement après leur arrivée en France, les Roumains décident de s'organiser et parfois même de reproduire des structures et des communautés connues dans leur pays d'origine: le Comité national roumain (CNR) et le Comité d'assistance aux Roumains (CAROMAN) fondés en 1947, le Conseil des Partis politiques qui réunit les partis politiques roumains dissous par le régime communiste de Bucarest (PNL, PNT, PSDI ${ }^{43}$ ), l'Union roumaino-française à Paris, l'Association culturelle Mihai Eminescu, etc. ${ }^{44}$. Les membres de l'exil réussissent ainsi à prouver que le régime de Bucarest «a supprimé toutes les formes de pluralisme politique », raison qui les a forcés à trouver refuge en Occident. Ils maintiennent donc l'idée d'une "Roumanie alternative, démocratique» représentée par les membres des anciens partis politiques historiques de Roumanie ${ }^{45}$.

Cependant, malgré ces exemples, jusque dans la seconde moitié des années 1970, l'exil roumain parisien est assez désorganisé. Les exilés agissent plutôt de manière individuelle ou dans des réseaux très restreints. Ils se mobilisent ensemble ponctuellement, lors des événements importants - comme au moment de la visite de Ceausescu en France (1970) - ou pour aider les nouveaux arrivants. Les Roumains en exil ne constituent pas une communauté homogène. Le caractère compliqué des relations interpersonnelles au sein de la diaspora mériterait d'ailleurs une analyse plus approfondie.

\footnotetext{
40 CNSAS, Virgil Ierunca, 349, note $n^{\circ}$ 141, 12 mai 1956, 1p ; F. Manolescu, Enciclopedia..., op. cit., p. 390-395.

41 Romulus Rusan (dir.), Anii 1973-1989 : Cronica unui sfarsit de sistem, Bucarest, Fundatia Academia Civica, 2003, p. 1026.

42 Virgil Tanase, Blandine Tézé-Delafon, Ma Roumanie : entretiens avec Blandine Tézé-Delafon, Paris, Éditions Ramsay-de ortanze-Médias, 1990, p. 150.

43 En 1947, les Parti national paysan (PNT) et le Parti national libéral (PNL sont dissouts par le régime communiste roumain. Le Parti social démocrate indépendant (PSDI) est crée en 1946 suite au refus d'une partie des membres du Parti social-démocrate (PSD) de s'unir avec le Parti des travailleurs roumains (PMR). Le PDSI est dissout à son tour en 1948 et ses dirigeants, comme ceux du PNT et du PNL sont également emprisonnés.

44 Gavin Bowd, La France et la Roumanie communiste, Paris, l’Harmattan, 2008, p. 106.

45 Dumitru Dobre, Iulia Huiu, Mihaela Toader, Sursele Securitatii informeaza, Bucarest, Humanitas, 2008, p. 23-24.
} 
Béatrice Scutaru, « La Roumanie à Paris : exil politique et lutte anti-communiste », Histoire@Politique. Politique, culture, société, n²3, mai-août 2014 [en ligne, www.histoire-politique.fr]

C'est seulement plus tard qu'ils commencent à mieux s'organiser et que leurs actions de dénonciation du régime roumain commencent à avoir plus d'impact. En effet, avant le milieu des années 1970, leurs actions ne sont pas couronnées de succès, les négociations et la signature de l'Acte final d'Helsinki changeant la donne. La signature et la publication de l'Acte final créent « le prémisse légal pour invoquer et sensibiliser l'opinion publique internationale sur le non respect des droits de l'homme par les États communistes 46 ». En fonction du public ciblé, les exilés mobilisent différents moyens d'action: édition de publications en roumain, participation à des émissions de radio et/ ou de télévision en direction de l'Occident et de la Roumanie, rédaction d'ouvrages historiques ou de romans, organisation de manifestations de protestation, envoi de rapports ou d'informations à la presse occidentale, envoi de protestations écrites aux gouvernements occidentaux et aux organismes internationaux, etc.

L'association qui a joué le rôle le plus important dans la dénonciation du régime roumain à partir de la fin des années 1970 est la Ligue pour la défense des droits de l'homme (LDHR) ${ }^{47}$. Constituée en 1977, elle a pour objectif de recueillir et de diffuser aux organisations internationales, aux gouvernements et aux médias des informations concernant les entorses aux droits de l'homme perpétrées en Roumanie. Elle souhaite montrer l'écart entre l'image favorable dont bénéficie cette démocratie populaire sur le plan international et les réalités de la politique menée sur le plan intérieur ${ }^{48}$. En 1982, la LDHR devient membre de la Fédération internationale des droits de l'homme (FIDH). La FIDH peut défendre les idées de la Ligue dans les organisations internationales, tout en donnant plus de légitimité aux critiques formulées contre le régime roumain ${ }^{49}$. Grâce à ces nouveaux types d'actions, la communauté réussit à acquérir une plus grande visibilité dans l'espace public national et international. La Ligue espère ainsi réussir à influer sur l'évolution du système international, qui ne peut pas être dissocié du combat pour la liberté de leur patrie $^{50}$.

La Ligue se mobilise en faveur des dissidents, des grévistes, des personnes persécutées pour leur foi religieuse, des prisonniers d'opinion, des membres du premier syndicat libre des travailleurs roumains, etc. En plus des cas très médiatisés, elle agit aussi pour aider des dizaines de personnes arrêtées, disparues, fusillées à la frontière yougoslave, la plupart du temps inconnues à l'Ouest de l'Europe et aux États-Unis. Des dossiers individuels ou thématiques sont envoyés régulièrement à Amnesty international, à la FIDH, aux gouvernements ou bien encore àla presse. La Ligue coopère avec l'organisation Helsinki Watch et avec la revue l'Alternative,

\footnotetext{
46Raluca Nicoleta Spiridon, « Reprimarea miscarilor muncitoresti de protest în perioada 1977-1987: evenimentele din Valea Jiului (1-3 august 1977) si revolta de la Brasov (15 noiembrie 1987) », dans Cosmin Budeanca, Florentin Olteanu (dir.), Forme de represiune în regimurile comuniste, Iasi, Polirom, 2008, p. 229.

${ }^{47} \mathrm{Au}$ début, l'association loi 1901 porte le nom de Comité pour la défense des droits de l'homme (CDHR) pour devenir une Ligue en 1979. Pour des soucis de clairette, seulement le terme de Ligue ou l'acronyme LDHR seront employés pour designer cette association.

48 Bibliothèque de documentation internationale contemporaine (BDIC), fonds LDHR, boîte $\mathrm{n}^{\circ} 1$ dossier $\mathrm{n}^{\circ}$ 4, statut de la Ligue pour la défense des droits de l'homme de Roumanie (LDHR), 1983, $5 \mathrm{p}$.

49 BDIC, LDHR, boîte $\mathrm{n}^{\circ} 2$, dossier $\mathrm{n}^{\circ} 1$, discours du président Constantin Cesianu à l'occasion de l'adhésion de la LDHR à la FIDH, Montréal, mai 1982, 4 p.

${ }^{50}$ Florence Vychytil-Baudoux, «'Le Comité électoral des Polonais naturalisés(1953-1976)'. Une expérience polonienne en France », Relations internationales, 2010/1, n 141, p. 69.
} 
Béatrice Scutaru, « La Roumanie à Paris : exil politique et lutte anti-communiste », Histoire@Politique. Politique, culture, société, n²3, mai-août 2014 [en ligne, www.histoire-politique.fr]

exclusivement consacrée aux pays socialistes. Elle participe également aux manifestations organisées par d'autres Comités des pays de l’Est, comme par exemple le Comité Solidarnosc ${ }^{51}$. Durant les années 1980, alors que la Roumanie et Nicolae Ceausescu sont de plus en plus critiqués, la Ligue acquiert un véritable statut sur la scène internationale. Les informations fournies par ses membres se retrouvent dans de nombreux articles de presse traitant de la Roumanie. De plus, les membres de la LDHR continuent la tradition initiée par la Fondation Charles I ${ }^{\mathrm{er}}$ : ils surveillent les parutions et les reportages sur la Roumanie et n'hésitent pas à faire pression sur les réalisateurs pour rectifier l'information ${ }^{52}$. Là où les actions de la Fondation n'avaient que rarement réussi, la Ligue a plus souvent gain de cause, tant en raison de la détérioration de l'image de la Roumanie que de la meilleure organisation des actions.

L'organisation et les moyens d'action des exilés évoluent sans cesse durant la période étudiée. Elles sont influencées et influencent à leur tour la société qui les accueille. C'est ce que Gabriel Scheffer appelle la« maturation diasporique ${ }^{53}$ ». Les Roumains qui ne souhaitent pas être assimilés par la société d'accueil - française - tentent de se positionner vis-à-vis de leur pays d'origine et s'organisent ainsi dans des structures qui leur permettent d'atteindre leurs objectifs. Tout au long de la période étudiée, leurs actions ont pour objectif de faire perdurer une tradition politique, culturelle...à l'Ouest, en attendant la chute du régime communiste et le retour au pays. Ceux qui décident de s'installer de l'autre côté du rideau de fer deviennent ainsi « les porteparole» des Roumains qui n'ont pas pu quitter le pays. Les exilés politiques se sentent investis du devoir d'informer, de dévoiler le véritable visage du régime de Bucarest. En effet, la question de la perception - de l'image - est un élément retrouvé en filigrane tout au long de cette étude. Elle joue un rôle déterminant dans les relations franco-roumaines durant la guerre froide. Un lien d'interdépendance peut même être établi entre l'évolution des relations bilatérales et la question de l'image. En effet, pendant la période communiste, les relations entre les Français et les Roumains ont connu leur meilleur développement quand l'image de la Roumanie de Ceausescu était à son apogée.

Cette volonté d'informer rend possible une circulation des personnes et des idées. En effet, outre leur rôle dans la dénonciation du régime Ceausescu, les exilés roumains réfugiés en France se font le relais des productions culturelles roumaines dans l'Hexagone. Ils font découvrir aux Français des publications, des idées que Bucarest ne souhaite pas voir diffusées à l'Ouest. Ils se font les vecteurs de transferts culturels entre la France et la Roumanie. Cette action ne peut et ne doit pas être limitée à la seule période de la guerre froide. À la suite de la chute du régime communiste, certains exilés retournent en Roumanie et jouent un rôle important dans la construction du pays et dans son processus d'européanisation. Cette étude, complétée avec une analyse similaire traitant de la période précédant la guerre froide, permettrait d'identifier les évolutions dans les pratiques politiques, économiques,

\footnotetext{
51 IICCMER, Sanda Stolojan, Liga pentru Apararea Drepturilor Omului in Romania de la Paris, 1983, 7p. 52 Béatrice Scutaru, « The Romanian Anti-Communist Resistance: the League for the Defence of Human Rights of Romania from Paris (1979-1989) », dans Peter Jasek (dir.), Anti-Communist Resistance..., op. cit., p. 842-843.

53 Gabriel Scheffer, Diaspora Politics. At Home Abroad, Cambridge, Cambridge University Press, 2003, p. 141.
} 
Béatrice Scutaru, « La Roumanie à Paris : exil politique et lutte anti-communiste », Histoire@Politique. Politique, culture, société, n²3, mai-août 2014 [en ligne, www.histoire-politique.fr]

sociales, littéraires... générées par ce double déracinement et les déplacements de l'exil politique.

\section{L'auteur}

Béatrice Scutaru, docteure en histoire de l'université d'Angers et chercheuse associée du CERHIO, a notamment publié des articles portant sur les échanges francoroumains durant la guerre froide.

\section{Résumé}

L'avènement du communisme détermine de nombreux Roumains à choisir la voie de l'exil, de la « liberté ». Les vagues d'émigrés se mobilisent et s'organisent pour recréer une société en exil, pour lutter contre le régime de Bucarest. L'exil roumain à Paris crée des associations politiques et culturelles, publie des revues et des romans et tente d'influer sur l'image de la Roumanie en France. L'objectif de cette étude est d'illustrer, à travers quelques exemples, l'importance de l'exil politique roumain dans la lutte anti-communiste.

Mots clés : exil ; anti-communisme ; guerre froide ; Roumanie ; Paris.

\section{Abstract}

The instauration of communism forced many Romanians to choose the path of exile, to choose "freedom". The multiple waves of émigrés mobilised and organised themselves in order to recreate their home society abroad, in order to fight against the Romanian communist regime. Romanian exiled in Paris created cultural and political organisations, published newspapers and novels and tried to change their home country's image abroad. This study argues that the Romanian political exile activities played an important part in the fight against communism.

Key words: exile, anti-communism, Cold War, Romania, Paris.

Pour citer cet article : Béatrice Scutaru, «La Roumanie à Paris : exil politique et lutte anti-communiste», Histoire@Politique. Politique, culture, société, n² 23, maiaoût 2014 [en ligne, www.histoire-politique.fr] 